\title{
A Deep Learning Classifier for Accurate Detection of the Novel Corona Virus
}

\author{
Cynthia Jayapa ${ }^{1 *}$, M. Sathiya Prakash² and M. Shiddharth Saran ${ }^{3}$ \\ ${ }^{1}$ Professor, Department of Computer Science and Engineering, \\ ${ }^{2,3}$ UG Scholars, Department of Computer Science and Engineering, \\ Kumaraguru College of Technology, Coimbatore, India
}

\section{ABSTRACT}

Although India witnessed the second slowest 100 to 1000 jump in COVID-19 cases, according to WHO, the number may be inaccurate because of the lack of rapid and large-scale testing facilities. According to reports, India is yet to face the gruesome effects of this pandemic as it moves closer to stage 4 of the community spread. Though standardized tests used in detecting coronaviruses, such as RT- PCR or transcriptase-polymerase chain reaction, take a minimum of 24 hours to generate useful results, they are also prone to high false negatives. Consequently, multiple periodic tests are required to arrive at a firm confirmation. Owing to this gap in the Indian Coronavirus testing scenario, this study focuses on a comparatively rapid and accurate method of testing employing AI-based image analysis of X-Ray and CT scans of the Lungs. Artificial intelligence based deep learning methodologies involving Convolutional Neural Networks with a sharp eye on accuracy of results and practical usage could be used for image analysis. Pre-trained and well-known convolutional neural networks along with a standard dataset for training and testing the same have been selected for the process. The performance of the model is also analyzed using standardized convolutional neural network analysis techniques to infer the best model for the particular use-case. The main objective of the study is to evaluate whether deep learning has the potential to provide accurate results and could provide aid to the existing X-ray methodology.

KEY WORDS: CONVOLUTIONAL NEURAL NETWORKS, COVID-19, DEEP LEARNING, IMAGE ANALYSIS.

\section{INTRODUCTION}

Coronavirus (COVID-19) has produced rampant unprecedented decimation with millions of people losing their lives (Sohrabi, C. et al., 2020). Being a member of the SARS family of viruses, which surfaced in the early 2000s, the COVID - 19 strain has been particularly deadly. Claimed to be originated in Wuhan (China), this strain has affected 214 countries and territories around

\section{ARTICLE INFORMATION}

*Corresponding Author: cynthia.j.it@kct.ac.in

Received 10th Oct 2020 Accepted after revision 25th Nov 2020

Print ISSN: 0974-6455 Online ISSN: 2321-4007 CODEN: BBRCBA

Thomson Reuters ISI Web of Science Clarivate Analytics USA and Crossref Indexed Journal

\section{Clarivate
Analytics}

NAAS Journal Score 2020 (4.31) SJIF: 2020 (7.728)

A Society of Science and Nature Publication,

Bhopal India 2020. All rights reserved.

Online Contents Available at: http//www.bbrc.in/

Doi: $h t t p: / / d x$.doi.org/10.21786/bbrc/13.11/1 the world along with two international conveyances. As per a publication by the infamous John Hopkins University, under extreme conditions, up to $10 \%$ of the Indian population could be susceptible to getting affected by COVID-19. This puts things into perspective as to why India is in dire need to contain the pandemic before it reaches stage 4 .

Thus, to achieve the same, we need rapid and accurate testing methods, contrary to the current time-intensive and inefficient methods (Yang, W. Yan, F. Patients et al.,2020) (Fang, Y. et al., 2020) (Xie, X. et al.,2020) to identify and isolate potential cases. From identifying the Zeroth patient in January to the current status(September 10,2020 ), India has seen a 6000-fold rise in COVID-19 cases and they still continue to grow linearly. This number has been reached by testing only through methods such as swab tests and blood tests. With a population of

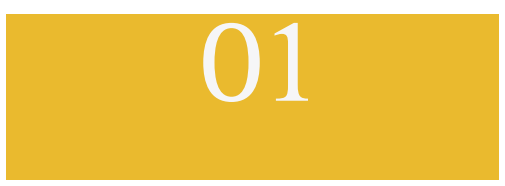


135 crores, studies suggest that up to 10\% of India's population ( 13.5 crores) are prone to COVID-19. The key is to test as many potential cases as possible. The present test which is done for the population rate is 18:1000000 which is too low because of the lack of kits.

Radiologists who deal with the diagnosis of the disease have observed common characteristics of physical conditions in the affected patients. The prominent method that is used to scan the affected body such as fractures, bone dislocations, lung infections, pneumonia, and tumors are X-ray machines. A computerized tomography (CT) scan (Yu, M. et al.,2020) combines a series of X-ray images taken from different angles around the body and uses computer processing to create crosssectional images (slices) of the bones, blood vessels and soft tissues inside your body. Using X-rays (Gomez P et al.,2019) is a faster, easier, cheaper, and less harmful method compared to CT scans. The proposed solution is implemented by classifying based on X-rays images. Since X-ray machines and diagnostic centers are in comfortably higher numbers and can test at the cost of less than INR 400 per test, also being reusable, unlike the existing testing methodologies. Thus the serviceable addressable market for this solution would be the people who have limited access to present testing methods and have the symptoms of COVID- 19 .

This could be employed to perform periodic tests for frontline workers involved in this fight against the pandemic and post check-up for COVID-19 survivors. Keeping in mind the present state of the outbreak and crisis involved, saving lives is more important though the solution has high monetizability. This method could also serve as a preliminary check for potential COVID19 patients before performing the actual swab/blood test which is limited in number. Thus we can save more PCR testing kits for more potential and vulnerable high risk patients. This method could highly improve the throughput of the patients checked everyday and thus, help us in isolating them and containing the outbreak. Therefore, this study aims at providing assistance to the existing X-ray methodology (Huang C et al.,2020) by proposing an accurate detection system for effective diagnosis in these tested times.

Since the problem scope addresses a classification problem(classifying people as either COVID-19 affected or not), the prominent approach to solving this could be done using classic AI. Artificial Intelligence (AI) (Negassi M et al.,2020) based automated CT and X-Ray image analysis tools will be of prominent use in the detection, quantification, and monitoring of the novel coronavirus. This method will effectively aid in distinguishing patients with coronavirus from benign ones. Based on the COVID-19 radiographic changes from CT images, advanced deep learning methods (Ching T et al.,2020) could extract the graphical features of COVID-19 induced changes in the Lungs. This provides clinical diagnosis prior to pathogenic testing and thus saves critical time in disease diagnosis. Deep learning is a subset of artificial intelligence that uses algorithmic structures called neural networks to perform classification tasks.

\section{MATERIAL AND METHODS}

Owing to its effective tunnel-shaped approach to network building, Convolutional neural networks are a patent choice for image classification. For this study, a particular variation of CNN based on pre-trained models(Huang, G. et al.,2016) that include ResNet50,VGG-16, and VGG19 was trained on a dataset consisting of chest X-ray radiographs. Using this classifier, hospitals could classify the severity and prevent many COVID-19 fatalities by preparing earlier by doing whatever they can to stop the virus before their symptoms get too serious. A total of 400 CT scan images were collected from the LIDC opensource public dataset. (Armato et al.,2011) The functional steps of the classifier are as follows:

- When the CNN based classifier takes an X-ray radiograph as input, the slices are first passed through a feature extractor based on a preprocessed ResNet50, VGG16, and VGG19. Given the slices per image, the result is a tensor of shape (s, 256, $7,7)$ for all the preprocessed models. Note: (256, 7,7 ) is simply a set of 256 features maps of size $7 \times 7$ obtained by the last convolutional layer of the model.

- $\quad$ Each tensor of shape, for example- $(256,7,7)$, is then reduced in a vector of sh (256,) by computing the mean value of each $7 \times 7$ square by global average pooling. The shape of the output is $(s, 256)$.

- In order to turn the (s, 256)-shaped tensor into a column vector before passing it to the classification part, max pooling is applied across the slices. A vector of shape (256, ). is obtained.

- A column vector that is obtained is passed through fully connected layers with Softmax activation functions and dropout.

- The exact form of a datapoint varies between tasks, it could be a single image, a slice of a time series, a tabular record, or something else entirely. These are then passed on to a data loader that handles batching of data points and parallelism.

- A series of geometric transformations are carried out on each input of the radiographic image.

- The transformations are label-invariant and are meant to bring diversity to the dataset thereby increasing the stability of the model while decreasing its tendency to overfitting.

- Three geometric transformations are sequentially applied on each input image.

- Random rotation between -25 and 25 degrees is 
applied.

- The random shift in both direction between -25 and 25 pixels

- Random horizontal flip with 50 percent probability

- The data obtained is then used to incrementally improve the model's ability to further classify the images.

- Random weights and biases are assigned during training the model. The results come out pretty poorly. The weights and biases are subsequently adjusted so as to obtain more correct predictions.

- The training of the model is done through the minimization of the cross-entropy loss using Adam optimizer.

\section{RESULTS AND DISCUSSION}

Figure 1: VGG -16 parameters

Training Loss and Accuracy on COVID-19 Dataset Using VGG1

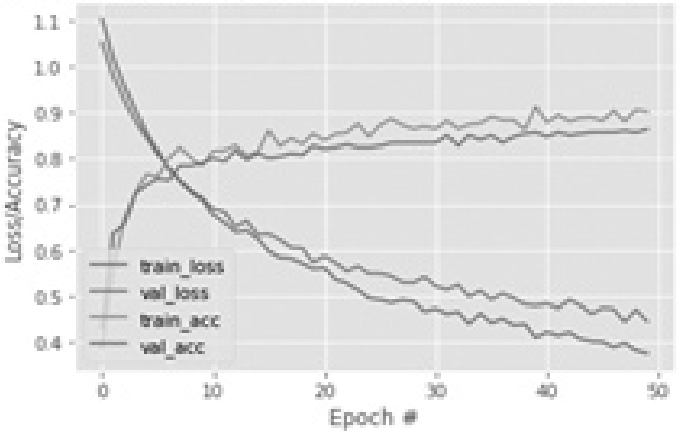

Figure 2: VGG -19 parameters

Training Loss and Accuracy on COVID-19 Dataset Using VGG19 CNN

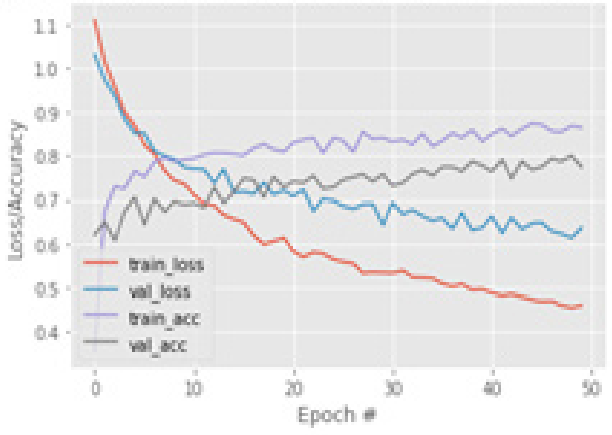

Figure 3: ResNet50 parameters

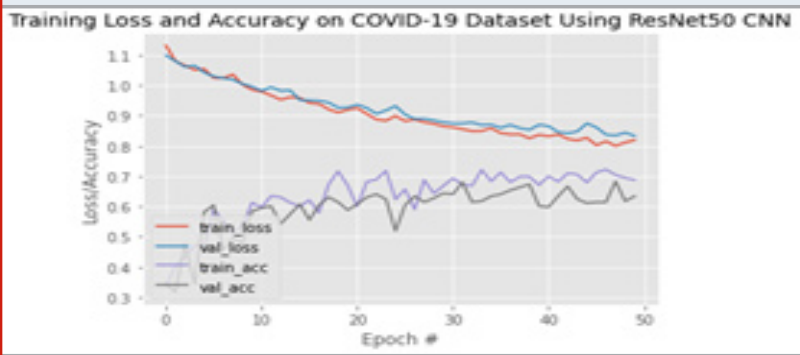

The models were subjected to Precision-Recall and confusion matrices analysis. The following results were inferred. (Refer Table 1)

The classifier was built with the best image classification deep neural networks with the most valid parameters to test them against. It can also be inferred that the chest X-ray images are better suited than the current methods for the detection of novel coronavirus. It is also observed that the pre-trained VGG16 model (Refer Figure 1) provides a high classification performance with a validation accuracy of 87\% followed by VGG-19 (Refer Figure 2) and ResNet50 (Refer Figure 3)

As the COVID-19 cases keep rising, our country is nowhere near flattening the curve. Through this proposed system, as a supplement to the existing X-ray methodology, we could improve the diagnostic accuracy, specificity, and also reduce the diagnostic time required for the clinical experts. With the proposed system's accuracy, it could effectively replace the current timeintensive methods thereby better equipping India in this fight against COVID-19.

Table 1. Classification Analysis

\begin{tabular}{|c|c|c|c|c|}
\hline \multirow{2}{*}{ MODEL } & & Precision & Recall & F1-score \\
\hline \multirow{2}{*}{ VGG -16 } & Covid-19 & 0.35 & 0.34 & 0.35 \\
\cline { 2 - 5 } & Normal & 0.38 & 0.52 & 0.44 \\
\hline \multirow{2}{*}{ VGG-19 } & Covid-19 & 0.31 & 0.22 & 0.25 \\
\cline { 2 - 5 } & Normal & 0.38 & 0.52 & 0.44 \\
\hline \multirow{2}{*}{ ResNet50 } & Covid-19 & 0.33 & 0.49 & 0.39 \\
\cline { 2 - 5 } & Normal & 0.30 & 0.41 & 0.35 \\
\hline
\end{tabular}

\section{REFERENCES}

Armato, S. G. 3rd et al. The lung image database consortium (LIDC) and image database resource initiative (IDRI): a completed reference database of lung nodules on CT scans. Med. Phys. 38, 915-931 (2011)

Ching T, Himmelstein DS, Beaulieu- Jones BK, Kalinin AA, Do BT, Way GP, et al. Opportunities and obstacles for deep learning in biology and medicine. J R Soc Interface. 2018;15(141):20170387. doi:10.1098/ rsif.2017.0387 (2018)

Fang, Y. et al. Sensitivity of chest CT for COVID19: comparison to RT-PCR. Radiology. https://doi. org/10.1148/ radiol.2020200432 (2020)

Gomez P, Semmler M, Schutzenberger A, Bohr C, Dollinger M. Low-light image enhancement of highspeed endoscopic videos using a convolutional neural network. Med Biol Eng Comput. (2019)

Huang, G., Liu, Z., van der Maaten, L. Weinberger, K. Q. Densely connected convolutional networks (2016). 
Huang C, Wang Y, Li X, Ren L, Zhao J, Hu Y, et al. Clinical features of patients infected with 2019 novel coronavirus in Wuhan, China. Lancet. (2020)

Negassi M, Suarez-Ibarrola R, Hein S, Miernik A, Reiterer A. Application of artificial neural networks for automated analysis of cystoscopic images: a review of the current status and future prospects. World J Urol. (2020)

Sohrabi, C. et al. World Health Organiza- ion declares global emergency: a review of the 2019 novel coronavirus (COVID-19). Int J. Surg. 76, 71-76 (2020)
Xie, X. et al. Chest CT for typical 2019-nCoV pneumonia:relationship to negative RT-PCR testing. Radiology. https://doi.org/10.1148/ radiol.2020200343 (2020).

Yang, W. Yan, F. Patients with RT-PCR-confirmed COVID-19 and normal chest CT. Radiology 295, E3 (2020).

$\mathrm{Yu}, \mathrm{M}$. et al. Thin-section chest CT imaging of coronavirus disease 2019 pneumonia: comparison between patients with mild and severe disease. Radiol. Cardiothorac. Imaging 2 https:// doi.org/10.1148/ ryct.2020200126 (2020). 